\title{
On the Construction of Pullbacks for Safe Petri Nets
}

\author{
Eric Fabre \\ IRISA/INRIA \\ Campus de Beaulieu \\ 35042 Rennes cedex, France \\ Eric.Fabre@irisa.fr
}

\begin{abstract}
The product of safe Petri nets is a well known operation: it generalizes to concurrent systems the usual synchronous product of automata. In this paper, we consider a more general way of combining nets, called a pullback. The pullback operation generalizes the product to nets which interact both by synchronized transitions and/or by a shared sub-net (i.e. shared places and transitions). To obtain all pullbacks, we actually show that all equalizers can be defined in the category of safe nets. Combined to the known existence of products in this category, this gives more than what we need: we actually obtain that all small limits exist, i.e. that safe nets form a complete category.
\end{abstract}

\section{Introduction}

We consider the category Nets of safe Petri nets (PN) as defined by Winskel in [2]. Safe Petri nets provide a natural and widespread model for concurrent systems. A product $\times$ was defined in 2 for safe PNs, that can be considered as a generalization of the usual synchronous product of automata. In practice, this product is essentially interesting when specialized to labeled nets : roughly speaking, it would then synchronize transitions of two nets as soon as they carry the same label. It therefore offers a very natural way to build large concurrent systems from elementary components. As a nice property, $\times$ is the categorical product in Nets . Pushing forward this idea, it can be interesting to derive a notion of pullback for PNs. While the product assumes that nets interact through common events, the pullback goes further and also allows interactions by shared places and transitions. Pullbacks can be used, for example, to combine two concurrent systems that synchronize through common events and at the same time share some resources (e.g. locks to access data).

The notion of pullback has been extensively explored for other models of concurrency (transition graphs, graph grammars, etc.) 7], or for other categories of Petri nets [3] (proposition 11). But the choice of net morphisms plays a crucial role, and apparently the construction of pullbacks in the category Nets of 2] is still missing. This category remains of great interest however, because it allows foldings (and consequently unfoldings!), and already has a product. Unfoldings have become an important tool for the verification of concurrent systems $10,11,12,13,14,15$. They have also been advocated for the monitoring 
of concurrent systems [16]. In particular, this second application domain relies intensively on factorization properties of unfoldings : the fact that the unfolding of a product system can be expressed as a product of unfoldings of its components [17. This property is actually the key to distributed or modular monitoring algorithms (surprisingly, this approach has not been explored in model checking applications, to the knowledge of the author). The derivation of the factorization property on unfoldings (or on other structures like trellises [18, 19]) relies on categorical arguments, and in particular on the fact that the unfolding operation preserves limits, like the product for example. In order to obtain a similar property for other ways of combining components, it is therefore crucial to characterize them as categorical limits. This is the main motivation of the present work.

Let us mention some contributions to the topic. B. Koenig provides in [9] a definition for specific pullback diagrams. M. Bednarczyk et al. prove in [8] that Nets is finitely complete, so all pullbacks exist. But the result is obtained in a much more general setting, and is hard to specialize to the case of safe nets. Finally, let us stress that [8] mentions in its introduction (p.3) that the existence of a pullback construction for safe Petri nets has been reported... although the authors have not been able to locate any reference! It is therefore useful to provide a simple and direct definition for this construction.

We proceed in several steps. We first consider unlabeled nets. It is a well known fact that the labeling is essentially a decoration that can be reincorporated at no cost in net operations (see [5]), which we do at the end of the paper (section 44). Secondly, we recall (section 2) that a pullback operation can be derived from a product and an equalizer (see [1, chap. V-2, thm. 1, and 7], sec. 5). Since all products exist in Nets, we simplify the construction (and proofs) by building equalizers, which is the heart of the contribution (section 3). We finally gather all pieces to give a comprehensive definition of the pullback of labeled Petri nets (section 4), first in the general case, then in the specific case where morphisms are partial functions. The conclusion underlines some important consequences of this construction.

\section{Notations}

Net. We denote Petri nets by $\mathcal{N}=\left(P, T, \rightarrow, P^{0}\right)$, representing respectively places, transitions, initially marked places and the flow relation. For each place $p \in P$, we assume $\left|p^{\bullet} \cup^{\bullet} p\right| \geq 1$, and for each transition $t \in T,\left|t^{\bullet}\right| \geq 1$ and $|\bullet t| \geq 1$. For labeled nets, we take $\mathcal{N}=\left(P, T, \rightarrow, P^{0}, \lambda, \Lambda\right)$ where $\lambda: T \rightarrow \Lambda$ is the labeling function.

Morphism. A morphism [2] $\phi: \mathcal{N}_{1} \rightarrow \mathcal{N}_{2}$ between nets $\mathcal{N}_{i}=\left(P_{i}, T_{i}, \rightarrow_{i}, P_{i}^{0}\right)$ is a pair $\left(\phi_{P}, \phi_{T}\right)$ where

C1. $\phi_{T}: T_{1} \rightarrow T_{2}$ is a partial function, and $\phi_{P}$ a relation between $P_{1}$ and $P_{2}$,

C2. $P_{2}^{0}=\phi_{P}\left(P_{1}^{0}\right)$ and $\forall p_{2} \in P_{2}^{0}, \exists$ a unique $p_{1} \in P_{1}^{0}: p_{1} \stackrel{\phi_{P}}{\longleftrightarrow} p_{2}$, 
C3. if $p_{1} \stackrel{\phi_{P}}{\longrightarrow} p_{2}$ then the restrictions $\phi_{T}:{ }^{\bullet} p_{1} \rightarrow{ }^{\bullet} p_{2}$ and $\phi_{T}: p_{1} \bullet \rightarrow p_{2} \bullet$ are total functions,

C4. if $t_{2}=\phi_{T}\left(t_{1}\right)$ then the restrictions $\phi_{P}^{o p}:{ }^{\bullet} t_{2} \rightarrow^{\bullet} t_{1}$ and $\phi_{P}^{o p}: t_{2}{ }^{\bullet} \rightarrow t_{1} \bullet$ are total functions.

where $\phi_{P}^{o p}$ denotes the opposite relation to $\phi_{P}$. Observe that condition C3 implies that if $\phi_{P}$ is defined at $p_{1} \in P_{1}$, then $\phi_{T}$ is defined at all transitions $t_{1} \in T_{1}$ connected to $p_{1}$. In the sequel, we will simply write $\phi$ for $\phi_{P}$ or $\phi_{T}$, and $\phi(X)$ to denote places in relation with at least one place in $X$. By $\operatorname{Dom}(\phi)$, we represent the elements of $\mathcal{N}_{1}$ (places or transitions) where $\phi$ is defined, i.e. $\phi^{o p}\left(P_{2} \cup T_{2}\right)$.

Notice that condition C3 entails that the pair $\left(\phi_{P}, \phi_{T}\right)$ preserves the flow relation (on its domain of definition). Together with $\mathrm{C} 4$ and $\mathrm{C} 2$, this guarantees that a run of $\mathcal{N}_{1}$ is mapped into a run of $\mathcal{N}_{2}$ by $\phi_{T}$ (see [2]), which is the least one should require from net morphisms. Simpler definitions of net morphisms would ensure this property, but C1-C4 are actually necessary to provide extra categorical properties, as we shall see in the sequel.

Remark. Notice that condition C2 becomes a consequence of C3 and C4 when one assumes the existence of a fake initial transition $t_{i, 0}$ in each $\mathcal{N}_{i}$, fed with a fake initial place $p_{i, 0} \rightarrow_{i} t_{i, 0}$, such that $t_{i, 0} \cdot=P_{i}^{0}$ and $t_{2,0}=\phi\left(t_{1,0}\right), p_{2,0} \stackrel{\phi}{\longleftrightarrow} p_{1,0}$. We shall use this trick in the sequel to simplify proofs (focusing on C3, C4 and omitting to check $\mathrm{C} 2$ ).

Safe Petri nets with the above definition of morphisms define the category Nets [2,4]. For labeled nets, we naturally consider label-preserving morphisms to define the category $\lambda$ Nets. Section 4 will detail the definition of this category.

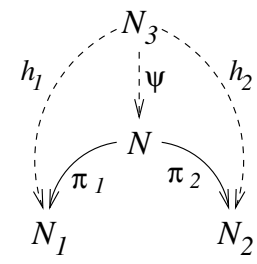

Fig. 1. Commutative diagram of the product $\mathcal{N}=\mathcal{N}_{1} \times \mathcal{N}_{2}$

Product. Let $\mathcal{N}_{1}, \mathcal{N}_{2}$ be nets, their categorical product $\mathcal{N}_{1} \times \mathcal{N}_{2}$ in Nets is a net $\mathcal{N}$ associated to morphisms $\pi_{i}: \mathcal{N} \rightarrow \mathcal{N}_{i}, i=1,2$, satisfying the so-called universal property of the product (fig. 1) : for every other candidate triple $\left(\mathcal{N}_{3}, h_{1}, h_{2}\right)$ with $h_{i}: \mathcal{N}_{3} \rightarrow \mathcal{N}_{i}$, there exists a unique morphism $\psi: \mathcal{N}_{3} \rightarrow \mathcal{N}$ such that $h_{i}=\pi_{i} \circ \psi$. This net $\mathcal{N}=\left(P, T, \rightarrow, P^{0}\right)$ and the $\pi_{i}$ are given by [4, 6]

1. $P=\left\{\left(p_{1}, \star\right): p_{1} \in P_{1}\right\} \cup\left\{\left(\star, p_{2}\right): p_{2} \in P_{2}\right\}$ : disjoint union of places, $\pi_{i}\left(p_{1}, p_{2}\right)=p_{i}$ if $p_{i} \neq \star$ and is undefined otherwise,

2. $P^{0}=\pi_{1}^{-1}\left(P_{1}^{0}\right) \cup \pi_{2}^{-1}\left(P_{2}^{0}\right)$,

3. $T=\left(T_{1} \times\{\star\}\right) \cup\left(\{\star\} \times T_{2}\right) \cup\left(T_{1} \times T_{2}\right), \quad \pi_{i}\left(t_{1}, t_{2}\right)=t_{i}$ if $t_{i} \neq \star$ and is undefined otherwise, 


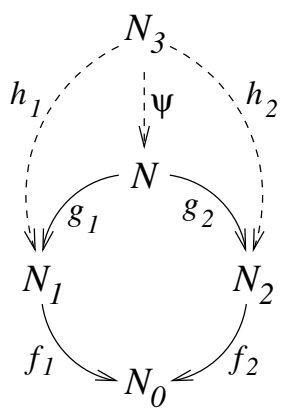

Fig. 2. Commutative diagram of the pullback $\mathcal{N}=\mathcal{N}_{1} \wedge \mathcal{N}_{2}$

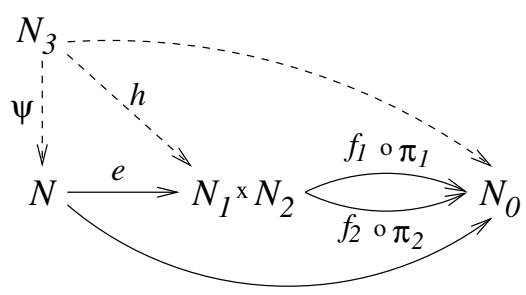

Fig. 3. Equalizing $f_{1} \circ \pi_{1}$ and $f_{2} \circ \pi_{2}$

4. the flow $\rightarrow$ is defined as follows: for $t \in T, \bullet t=\pi_{1}^{-1}\left(\bullet \pi_{1}(t)\right) \cup \pi_{2}^{-1}\left({ }^{\bullet} \pi_{2}(t)\right)$ and symm. for $t^{\bullet}$, assuming ${ }^{\bullet} \pi_{i}(t)=\pi_{i}(t)^{\bullet}=\emptyset$ if $\pi_{i}$ is undefined at $t$.

At first sight, this categorical product may look useless since every transition is free to fire alone or jointly with any transition of the other net. Again, the interest of this construction appears when it is applied to labeled nets, in association with a synchronization algebra 4. Its practical interest then becomes obvious to build large systems starting from elementary components. Since labels bring no technical difficulty other than notational, we put them aside until section 4 .

Decomposition of the pullback. Let $\mathcal{N}_{0}, \mathcal{N}_{1}, \mathcal{N}_{2}$ be nets, and $f_{i}: \mathcal{N}_{i} \rightarrow \mathcal{N}_{0}, i=1,2$ be net morphisms, so $\mathcal{N}_{0}$ forms a kind of interface between $\mathcal{N}_{1}$ and $\mathcal{N}_{2}$. We look for a terminal net $\mathcal{N}=\left(P, T, \rightarrow, P^{0}\right)$, associated to morphisms $g_{i}: \mathcal{N} \rightarrow \mathcal{N}_{i}$, $i=1,2$, such that (fig. 20) :

$$
f_{1} \circ g_{1}=f_{2} \circ g_{2}
$$

By "terminal," we mean the universal property of the pullback: whenever there exists another triple $\left(\mathcal{N}_{3}, h_{1}, h_{2}\right)$ satisfying the same commutative diagram, there exists a unique mediating morphism $\psi: \mathcal{N}_{3} \rightarrow \mathcal{N}$ such that $h_{i}=g_{i} \circ \psi$. We denote the pullback by $\mathcal{N}_{1} \wedge \mathcal{N}_{0} \mathcal{N}_{2}$, or by $\mathcal{N}_{1} \wedge \mathcal{N}_{2}$ for short.

It is well known that the pullback operation can be decomposed into a product, followed by an equalization. Consider the product net $\mathcal{N}_{1} \times \mathcal{N}_{2}$, and the associated canonical projections $\pi_{i}: \mathcal{N}_{1} \times \mathcal{N}_{2} \rightarrow \mathcal{N}_{i}, i=1,2$. In general, $\mathcal{N}_{1} \times \mathcal{N}_{2}$ and 
the $\pi_{i}$ do not satisfy the pullback condition, i.e. $f_{1} \circ \pi_{1} \neq f_{2} \circ \pi_{2}$. However, by equalizing them, one gets the desired result. $(\mathcal{N}, e)$ equalizes $f_{1} \circ \pi_{1}$ and $f_{2} \circ \pi_{2}$ iff $\left(f_{1} \circ \pi_{1}\right) \circ e=\left(f_{2} \circ \pi_{2}\right) \circ e$, and for any other candidate $\left(\mathcal{N}_{3}, h\right)$ there exists a unique $\psi: \mathcal{N}_{3} \rightarrow \mathcal{N}$ such that $h=e \circ \psi$ (fig. 3). It is straightforward to check that $\left(\mathcal{N}, \pi_{1} \circ e, \pi_{2} \circ e\right)$ then yields the desired pullback. For details, we refer the reader to [1], chap. V-2, thm. 1, or to 7], sec. 5 where this construction is also used.

\section{Equalizer in Nets}

Consider two nets $\mathcal{N}_{i}=\left(P_{i}, T_{i}, \rightarrow_{i}, P_{i}^{0}\right), i=1,2$ related by two morphisms $f, g: \mathcal{N}_{1} \rightarrow \mathcal{N}_{2}$. We want to build the equalizer $(\mathcal{N}, e)$ of $f$ and $g$, i.e. a net $\mathcal{N}$ and a morphism $e: \mathcal{N} \rightarrow \mathcal{N}_{1}$ satisfying $f \circ e=g \circ e$, and such that for any other candidate pair $\left(\mathcal{N}_{3}, h\right)$ there exists a unique morphism $\psi: \mathcal{N}_{3} \rightarrow \mathcal{N}$ satisfying $h=e \circ \psi$ (fig. 4).

\subsection{Equalizer and Coequalizer in Sets}

We recall here two classical results that will be instrumental in the sequel.

Equalizer. We consider the category of sets with partial functions as morphisms (or equivalently pointed sets with total functions). Let $T_{1}, T_{2}$ be two sets related by partial functions $f, g: T_{1} \rightarrow T_{2}$. The equalizer of $f$ and $g$ is the pair $(T, e)$ where

$$
T=\left\{t_{1} \in T_{1}: f\left(t_{1}\right)=g\left(t_{1}\right) \text { or both } f \text { and } g \text { are undefined at } t_{1}\right\}
$$

and $e$ is the canonical injection of $T$ into $T_{1}$ (we'll use the shorthand $t_{1} \in T$ instead of $\left.t \in T, t_{1}=e(t)\right)$. In the setting of pointed sets, where functions point to the special value $\epsilon$ of a set to mean "undefined," (2) takes the simplest form $f\left(t_{1}\right)=g\left(t_{1}\right)$.

Given another candidate pair $\left(T_{3}, h\right)$, the unique morphism (partial function) $\psi: T_{3} \rightarrow T$ is obtained by $\psi=e^{-1} \circ h$ (it is easy to check that $\operatorname{Im}(h) \subseteq T$ ).

Coequalizer. We now consider the category of sets with total functions. The coequalizer diagram corresponds to fig. 4 with all arrows reversed. Let $S_{2}, S_{1}$ be two sets related by total functions $F, G: S_{2} \rightarrow S_{1}$, and denote by $(S, E)$ the coequalizer of $F$ and $G$. The construction is a bit more complex.

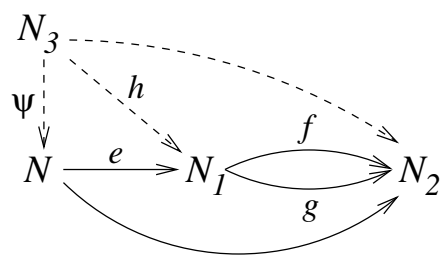

Fig. 4. A pair $(\mathcal{N}, e)$ equalizing $f$ and $g$ 


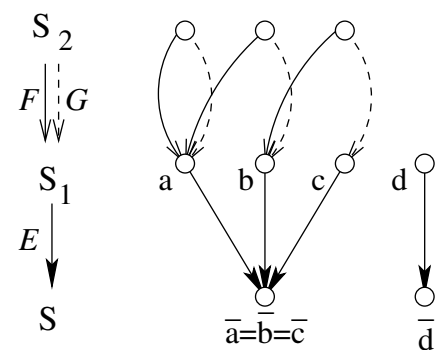

Fig. 5. Coequalizing the total functions $F$ and $G$

Define the relation $R$ on elements of $S_{1}$ by

$$
p_{1} R p_{1}^{\prime} \Leftrightarrow \exists p_{2} \in S_{2},\left\{p_{1}, p_{1}^{\prime}\right\}=\left\{F\left(p_{2}\right), G\left(p_{2}\right)\right\}
$$

and consider the equivalence relation $\equiv$ generated by $R$. We denote by $\left[p_{1}\right]$ the class of $p_{1}$ for $\equiv$. Then

$$
S=\left\{\left[p_{1}\right]: p_{1} \in S_{1}\right\}
$$

and the function $E: S_{1} \rightarrow S$ is simply the quotient operation, i.e. $E\left(p_{1}\right)=\left[p_{1}\right]$. See fig. $[5$ for an example.

Given another candidate pair $\left(S_{3}, H\right)$, the unique morphism (total function) $\Psi: S_{3} \rightarrow S$ is obtained by $\Psi=H \circ E^{-1}$, or in other words by $\forall\left[p_{1}\right] \in S, \Psi\left(\left[p_{1}\right]\right)=$ $H\left(p_{1}\right)$. Indeed, it is easy to check that $H$ is necessarily class invariant.

\subsection{Candidate Equalizer in Nets}

Let $(\mathcal{N}, e)$ denote the desired equalizer, with $\mathcal{N}=\left(P, T, \rightarrow, P^{0}\right)$ and $e: \mathcal{N} \rightarrow \mathcal{N}_{1}$.

Transitions. On transition sets, $f, g: T_{1} \rightarrow T_{2}$ are partial function, so we adopt definition (2) for $T$ and $e$ on $T$.

Places. On place sets, the definition is a bit more complex. The morphism definition in Nets actually states in C4 that $\phi^{o p}:{ }^{\bullet} t_{2} \rightarrow{ }^{\bullet} t_{1}$ and $\phi^{o p}: t_{2}{ }^{\bullet} \rightarrow t_{1} \bullet$ are total functions, for $t_{2}=\phi\left(t_{1}\right)$, which orients us to co-equalizers in Sets. So let $t$ be a transition of $T$, with $t_{1}=e(t) \in T_{1}$.

Assume first that $f, g$ are defined at $t_{1}$, and $f\left(t_{1}\right)=g\left(t_{1}\right)=t_{2} \in T_{2}$. We take for $e^{o p}$ in ${ }^{\bullet} t_{1}$ the coequalizer of $f^{o p}, g^{o p}:{ }^{\bullet} t_{2} \rightarrow{ }^{\bullet} t_{1}$. Eq. (3) thus defines $R^{\bullet t_{1}}$, the equivalence relation $\equiv^{t_{1}}$ and place classes $\left[p_{1}\right]^{\bullet} t_{1}$. And similarly in the post-set of $t_{1}$.

When $f, g$ are both undefined at $t_{1}$, we take for $e^{o p}$ in $\bullet_{1}$ (or $t_{1}^{\bullet}$ ) the coequalizer of functions $f^{o p}, g^{o p}$ from the empty set. So $e^{o p}$ is simply the identity.

In summary, the place set $P$ of $\mathcal{N}$ is a subset of $2^{P_{1}}$ given by

$$
P=\left\{\left[p_{1}\right]^{\bullet} t_{1}: t_{1} \in T, p_{1} \in t_{1}\right\} \cup\left\{\left[p_{1}\right]^{t_{1}}: t_{1} \in T, p_{1} \in t_{1} \bullet\right\}
$$

and the relation $e$ on places is simply given by $p \stackrel{e}{\longleftrightarrow} p_{1}$ iff $p_{1} \in p$. Observe that a place $p_{1} \in P_{1}$ not connected to a transition of $T$ has no counterpart in $P$. 


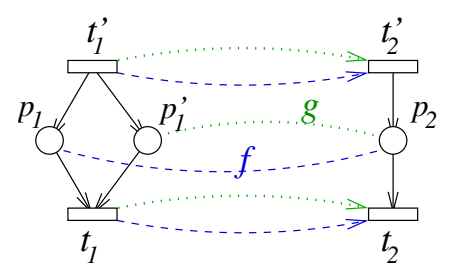

Fig. 6. Identity of equivalence classes

Lemma 1. Let $t_{1}, t_{1}^{\prime} \in T$. Assume $p_{1}, p_{1}^{\prime} \subseteq t_{1}^{\prime} \cap \bullet^{\bullet} t_{1}$, then

$$
p_{1} \equiv^{t_{1}^{\prime}} p_{1}^{\prime} \Longleftrightarrow p_{1} \equiv{ }^{\bullet} t_{1} p_{1}^{\prime}
$$

Proof. Assume $p_{1} \neq p_{1}^{\prime}$ and $p_{1} R_{1}^{t_{1}^{\bullet}} p_{1}^{\prime}$. This means $f, g$ are defined at $t_{1}^{\prime}, f\left(t_{1}^{\prime}\right)=$ $t_{2}^{\prime}=g\left(t_{1}^{\prime}\right)$, and for exampl迎 $\exists p_{2} \in t_{2}^{\prime} \bullet: p_{1} \stackrel{f}{\longleftrightarrow} p_{2} \stackrel{g}{\longleftrightarrow} p_{1}^{\prime}$. Let $t_{2}=f\left(t_{1}\right)=$ $g\left(t_{1}\right)$, by $\mathrm{C} 3$ on $f$ or $g$, one has $p_{2} \in{ }^{\bullet} t_{2}$, whence $p_{1} R^{\bullet} t_{1} p_{1}^{\prime}$. This proves $\left[p_{1}\right]^{t_{1}^{\bullet}} \subseteq$ $\left[p_{1}\right]^{\bullet} t_{1}$. One can show in the same way the reverse inclusion, which proves the lemma.

Naturally, the lemma holds also for the other arrow orientations, i.e. for $p_{1}, p_{1}^{\prime} \subseteq$ $t_{1}^{\prime} \cap t_{1}^{\bullet}$ and for $p_{1}, p_{1}^{\prime} \subseteq \bullet t_{1}^{\prime} \cap \bullet t_{1}$.

Initial places. In eq. (5), we assume the existence of (fake) transitions $t_{i, 0}$ with $t_{i, 0} \bullet=P_{i}^{0}$ and $f\left(t_{1,0}\right)=g\left(t_{1,0}\right)=t_{2,0}$. So initial places in $P$ are given by

$$
P^{0}=\left\{\left[p_{1}\right]^{t_{1,0}}: p_{1} \in P_{1}^{0}\right\}
$$

For $p_{1} \in P_{1}$ and $t_{1} \in T_{1}$, notice that the equivalence class $\left[p_{1}\right]^{\bullet} t_{1}$ (or equivalently $\left.\left[p_{1}\right]^{t_{1}}{ }^{\bullet}\right)$ may both contain marked places of $P_{1}^{0}$ and unmarked places of $P_{1} \backslash P_{1}^{0}$. Such a class is not taken as an initial place of $\mathcal{N}$. See the example of $p^{\prime}$ in fig. 7 .

Conversely, assume an equivalence class $\left[p_{1}\right]^{\bullet} t_{1}$ (for ex.) satisfies $\left[p_{1}\right]^{\bullet} t_{1} \subseteq P_{1}^{0}$. By lemma 1. $\left[p_{1}\right]^{\bullet} t_{1}=\left[p_{1}\right]^{t_{1}^{\bullet}}$ which corresponds to an initial place of $\mathcal{N}$. We could thus take as an alternate definition:

$$
P^{0}=\left\{p \in P: e(p) \subseteq P_{1}^{0}\right\}
$$

Flow relation. It is obviously defined by $p \rightarrow t$ when $e(t)=t_{1}$ and $p=\left[p_{1}\right]^{\bullet} t_{1}$ for some $p_{1} \in{ }^{\bullet} t_{1}$. But, using lemma 1, we can derive the simpler criterion:

$$
p \rightarrow t \Longleftrightarrow e(p) \subseteq \cdot e(t) \text { in } \mathcal{N}_{1}
$$

We proceed symmetrically for $t \rightarrow p$.

Example. Fig. 7 illustrates this construction. Observe that $p_{1} R^{t_{1}^{\prime}} p_{1}^{\prime}$ and $p_{1} R^{\bullet} t_{1}$ $p_{1}^{\prime \prime}$, which results in two classes/places in $\mathcal{N}$, both related to $p_{1}$ by $e$. These places must indeed be distinguished: by merging places $p^{\prime}$ and $p$ in $\mathcal{N}$, i.e. by aggregating classes sharing one or more places of $P_{1}$, the resulting $e$ wouldn't be a morphism (C3 violated).

\footnotetext{
${ }^{1}$ The other possibility is $p_{1} \stackrel{g}{\longleftrightarrow} p_{2} \stackrel{f}{\longleftrightarrow} p_{1}^{\prime}$, but this doesn't affect the proof.
} 


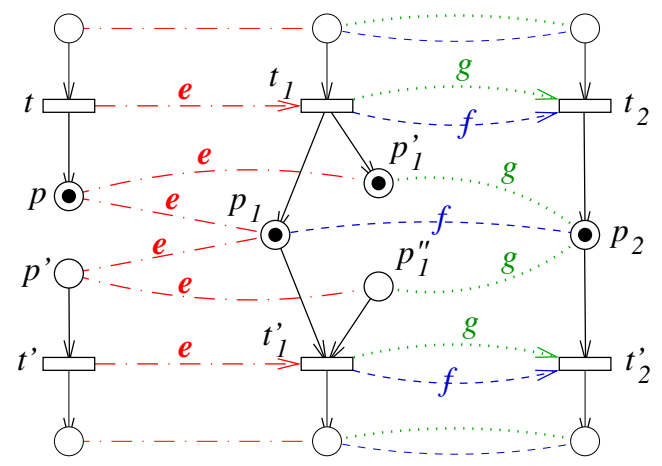

Fig. 7. The equalizer $(\mathcal{N}, e)$ (left) for nets $\mathcal{N}_{1}$ (center) and $\mathcal{N}_{2}$ (right) related by two morphisms $f, g$. Notice that $t^{\prime}, t_{1}^{\prime}, t_{2}^{\prime}$ could be the "fake" initial transitions.

\subsection{Coherence of the Definition}

$e: \mathcal{N} \rightarrow \mathcal{N}_{1}$ is a net morphism. C1 holds by definition, and with the trick of fake initial transitions, $\mathrm{C} 2$ is a consequence of $\mathrm{C} 3$ and $\mathrm{C} 4$, which we only need to examine.

C4 obviously holds by construction of places of $P$ : let $t_{1}=e(t)$, then $e^{o p}: \bullet^{\bullet} t_{1} \bullet^{\bullet} t$ defined by $e^{o p}\left(p_{1}\right)=\left[p_{1}\right]^{\bullet} t_{1}$ is a total function. And similarly for $e^{o p}: t_{1} \bullet \rightarrow t^{\bullet}$.

For $\mathrm{C} 3$, consider $p \rightarrow t$ in $\mathcal{N}$, such that $p \stackrel{e}{\longleftrightarrow} p_{1}$ and $e(t)=t_{1}$. We want to check that $p_{1} \rightarrow_{1} t_{1}$ in $\mathcal{N}_{1}$. By definition of the flow in $\mathcal{N}$, one has $p \rightarrow t$ iff $e(p) \subseteq \bullet e(t)=\bullet t_{1}$, and $p \stackrel{e}{\longleftrightarrow} p_{1}$ iff $p_{1} \in p$, so $p_{1} \rightarrow_{1} t_{1}$ holds. The same reasoning proves that $e: \bullet^{\bullet} p \rightarrow p_{1}$ is also a total function.

$\mathcal{N}$ is a safe net. By a standard argument 2]: since $e: \mathcal{N} \rightarrow \mathcal{N}_{1}$ is a net morphism, it maps runs of $\mathcal{N}$ to runs of $\mathcal{N}_{1}$. So if $\mathcal{N}$ is not safe, one of its run fills some place with more than one token, which reveals by $e$ a non safe run in $\mathcal{N}_{1}$, because $e$ is a total function on $T$.

$(\mathcal{N}, e)$ satisfies the commutative diagram. This is true by construction for the partial functions on transitions. It also holds locally for relations on places, i.e. around triples of transitions $\left(t, t_{1}, t_{2}\right)$ with $\left.t_{1}=e(t), t_{2}=f\left(t_{1}\right)=g\left(t_{1}\right)\right)$. This allows to reach completely the place relations $e, f, g$.

\subsection{Universal Property}

Assume the pair $\left(\mathcal{N}_{3}, h\right)$ satisfies $f \circ h=g \circ h$, with $N_{3}=\left(P_{3}, T_{3}, \rightarrow_{3}, P_{3}^{0}\right)$ and $h: \mathcal{N}_{3} \rightarrow \mathcal{N}_{1}$. We look for a (unique) $\psi: \mathcal{N}_{3} \rightarrow \mathcal{N}$ satisfying $h=e \circ \psi$ (see fig. (4).

Definition of $\psi$. On transitions, $\psi$ is uniquely given by $\psi=e^{-1} \circ h$, as it was seen in section 3.1 .

For places, consider a triple $\left(t_{3}, t, t_{1}\right) \in T_{3} \times T \times T_{1}$ of related transitions: $\psi\left(t_{3}\right)=t$ and $h\left(t_{3}\right)=t_{1}=e(t)$. We say that such a triple $\left(t_{3}, t, t_{1}\right)$ forms $a$ 
triangle. From the construction of co-equalizers in section 3.1, we know that $\psi^{o p}: \bullet t \rightarrow t_{3}$ is uniquely defined from $h^{o p}:{ }^{\bullet} t_{1} \rightarrow \bullet t_{3}$ by

$$
\forall p_{1} \in{ }^{\bullet} t_{1}, \quad \psi^{o p}\left([p 1]^{\bullet} t_{1}\right)=h^{o p}\left(p_{1}\right) \cap \bullet t_{3}
$$

Specifically, $h^{o p}\left(p_{1}\right) \cap \bullet t_{3}$ exists and is formed by a single place $p_{3}$ because $h$ is a net morphism and thus satisfies C4. Moreover, this value $p_{3}$ doesn't depend on the choice of $p_{1}$ in $[p 1]^{\bullet} t_{1}$ because, as a co-equalizer $h^{o p}$ is necessarily class invariant on ${ }^{\bullet} t_{1}$ (see 3.1). We proceed similarly to define $\psi^{o p}: t^{\bullet} \rightarrow t_{3}{ }^{\bullet}$.

$\psi$ satisfies the commutative diagram. By construction of $\psi, h=e \circ \psi$ is obvious on transitions, and locally on places (i.e. around triangles of transitions). To show that the relation holds globally on places, consider $p_{3} \in P_{3}$. By assumption, $p_{3}$ is connected to at least one transition $t_{3}$ in $\mathcal{N}_{3}$. If $h$ is defined at $p_{3}$ and $p_{3} \stackrel{h}{\longleftrightarrow} p_{1}$, then $h$ is also defined at $t_{3}$ (by C3), $h\left(t_{3}\right)=t_{1} \in T$ and $p_{1}$ is connected to $t_{1}$. We then use $h=e \circ \psi$ around the triangle $\left(t_{3}, t, t_{1}\right)$, where $t=\psi\left(t_{3}\right)$.

$\psi$ is a net morphism. It obviously satisfies $\mathrm{C} 1$, and $\mathrm{C} 4$ is imposed by the construction of $\psi$ on places. So only C3 has to be checked, which is the difficult part of the proof.

For C3, consider a pair of places $\left(p_{3}, p\right) \in P_{3} \times P$ related by $\psi\left(\right.$ i.e. $\left.p_{3} \stackrel{\psi}{\longleftrightarrow} p\right)$ and assume $p_{3} \rightarrow t_{3}$ in $\mathcal{N}_{3}$. We want to show that $\psi$ is defined at $t_{3}$, and $\psi\left(t_{3}\right) \in p^{\bullet}$ in $\mathcal{N}$. By definition of $\psi$ on places, there exists a triangle $\left(t_{3}^{\prime}, t^{\prime}, t_{1}^{\prime}\right) \in$ $T_{3} \times T \times T_{1}$ such that for example $t_{3}^{\prime} \rightarrow_{3} p_{3}, t_{1}^{\prime} \rightarrow_{1} p_{1}, t^{\prime} \rightarrow p$ and $p=\left[p_{1}\right]^{t_{1}^{\prime}}$ (see Fig. 8).

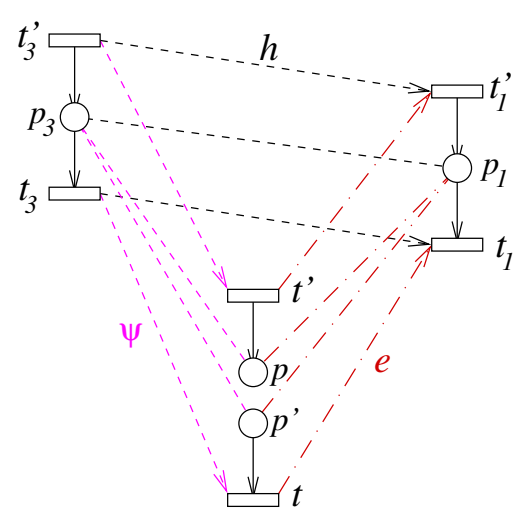

Fig. 8. Proof that $\psi$ satisfies C3

$h$ is defined at $p_{3}$, thus also at $t_{3}$ by C3. Since $f \circ h=g \circ h$, one has $t_{1}=$ $h\left(t_{3}\right) \in T$. So there exists $t \in T$ with $e(t)=t_{1}$ and thus we already know that $\psi$ is defined at $t_{3}: \psi\left(t_{3}\right)=t$. In other words, $\left(t_{3}, t, t_{1}\right) \in T_{3} \times T \times T_{1}$ forms another

${ }^{2}$ Equivalently, we could have assumed that the related places are in the presets (instead of post-sets) of a transition triangle. 
triangle. Since $e$ is a morphism, let $p^{\prime}$ be the image of $p_{1}$ by $e^{o p}:{ }^{\circ} t_{1} \rightarrow \bullet t$, so $p^{\prime}=\left[p_{1}\right]^{\bullet_{1}}$. By definition of $\psi$ in the presets of the triangle $\left(t_{3}, t, t_{1}\right)$, see (10), one has $p_{3} \stackrel{\psi}{\longleftrightarrow} p^{\prime}$. To conclude the proof, we thus have to show that $p=p^{\prime}$. We essentially use the fact that $h$ is a morphism satisfying $f \circ h=g \circ h$.

Let $p_{1}^{\prime}$ be a place of $t_{1}^{\prime \bullet}$ such that $p_{1} \equiv_{1}^{t^{\bullet}} p_{1}^{\prime}$. We know that $p_{3} \stackrel{h}{\longleftrightarrow} p_{1}^{\prime}$, because $h^{o p}: t_{1}^{\prime \bullet} \rightarrow t_{3}^{\prime \bullet}$ is class invariant (a consequence of $f \circ h=g \circ h$ ). From $p_{3} \rightarrow_{3} t_{3}$ in $\mathcal{N}_{3}$ and $p_{3} \stackrel{h}{\longleftrightarrow} p_{1}^{\prime}$, we derive by C3 that $p_{1}^{\prime} \rightarrow_{1} t_{1}=h\left(t^{\prime}\right)$. We are now exactly in the situation of lemma 1, so $p_{1} \equiv{ }^{{ }^{t}}{ }_{1} p_{1}^{\prime}$. We have thus proved that $\left[p_{1}\right]^{t_{1}^{\prime}}$ and $\left[p_{1}\right]^{\bullet_{1}}$ are identical, or in other words $p=p^{\prime}$.

\section{Application to Pullbacks of Labeled Nets}

We now reassemble all elements to provide a definition for pullbacks of safe labeled nets. The first task is to define the category $\lambda$ Nets . Consider labeled nets $\mathcal{N}_{i}=\left(P_{i}, T_{i}, \rightarrow_{i}, P_{i}^{0}, \lambda_{i}, \Lambda_{i}\right), \phi: \mathcal{N}_{1} \rightarrow \mathcal{N}_{2}$ is a morphism in $\lambda$ Nets iff $\phi$ is a net morphism (as defined in section 2 by $\mathrm{C} 1-\mathrm{C} 4$ ), with the extra requirements :

C5. $\phi_{T}$ preserves labels,

C6. $\Lambda_{1} \supseteq \Lambda_{2}$,

C7. $\operatorname{Dom}\left(\phi_{T}\right)=\lambda_{1}^{-1}\left(\Lambda_{2}\right)$.

The next section recalls the definition of the product in this category, that we combine to the equalizer to obtain the pullback.

\subsection{Product}

Let $\mathcal{N}_{i}=\left(P_{i}, T_{i}, \rightarrow_{i}, P_{i}^{0}, \Lambda_{i}, \lambda_{i}\right), i=1,2$ be two labeled nets. To build net products, we assume a simple synchronization algebra [5] : two transitions carrying the same label have to synchronize, while transitions carrying a private label remain private. Private labels are those in $\left(\Lambda_{1} \backslash \Lambda_{2}\right) \cup\left(\Lambda_{2} \backslash \Lambda_{1}\right)$. The product $\overline{\mathcal{N}}=\mathcal{N}_{1} \times \mathcal{N}_{2}$ and the associated projections $\pi_{i}: \overline{\mathcal{N}} \rightarrow \mathcal{N}_{i}$ are defined as follow: $3:$

1. $\bar{P}=\left\{\left(p_{1}, \star\right): p_{1} \in P_{1}\right\} \cup\left\{\left(\star, p_{2}\right): p_{2} \in P_{2}\right\}:$ disjoint union of places,

$\pi_{i}\left(p_{1}, p_{2}\right)=p_{i}$ if $p_{i} \neq \star$ and is undefined otherwise,

2. $\bar{P}^{0}=\pi_{1}^{-1}\left(P_{1}^{0}\right) \cup \pi_{2}^{-1}\left(P_{2}^{0}\right)$,

3 . the transition set $\bar{T}$ is given by

$$
\begin{aligned}
\bar{T} & =\left\{\left(t_{1}, \star\right): t_{1} \in T_{1}, \lambda_{1}\left(t_{1}\right) \in \Lambda_{1} \backslash \Lambda_{2}\right\} \\
& \cup\left\{\left(\star, t_{2}\right): t_{2} \in T_{2}, \lambda_{2}\left(t_{2}\right) \in \Lambda_{2} \backslash \Lambda_{1}\right\} \\
& \cup\left\{\left(t_{1}, t_{2}\right) \in T_{1} \times T_{2}: \lambda_{1}\left(t_{1}\right)=\lambda_{2}\left(t_{2}\right) \in \Lambda_{1} \cap \Lambda_{2}\right\}
\end{aligned}
$$

$\pi_{i}\left(t_{1}, t_{2}\right)=t_{i}$ if $t_{i} \neq \star$ and is undefined otherwise,

${ }^{3}$ Remark: if ones wishes to use the trick of fake initial transitions $t_{i}^{0}$ to define initial markings $P_{i}^{0}$ by $P_{i}^{0}=t_{i}^{0}$, one has to assume that each $\Lambda_{i}$ contains a special label $\epsilon^{0}$ reserved to the transition $t_{i}^{0}$. 
4. the flow $\rightarrow$ is defined by $\bullet t=\pi_{1}^{-1}\left(\bullet \pi_{1}(t)\right) \cup \pi_{2}^{-1}\left(\bullet \pi_{2}(t)\right)$ and symm. for $t^{\bullet}$, assuming $\bullet \pi_{i}(t)=\pi_{i}(t)^{\bullet}=\emptyset$ if $\pi_{i}$ is undefined at $t$,

5. $\bar{\Lambda}=\Lambda_{1} \cup \Lambda_{2}$ and $\bar{\lambda}$ is the unique labeling preserved by the $\pi_{i}$.

Let us recall that the product of labeled nets can also be obtained by taking the product of non-labeled nets, and then discarding transition pairs that violate the rules of the synchronization algebra.

For our choice of morphisms, it is straightforward to check that the above definition actually yields the categorical product in $\lambda$ Nets : The $\pi_{i}$ are net morphisms that obviously satisfy C5-C7. And for the universal property, with notations of fig. 1, the $\psi$ computed in Nets (ignoring labels) is defined by 4 $\forall t_{3} \in T_{3}, \psi\left(t_{3}\right)=\left(h_{1}\left(t_{3}\right), h_{2}\left(t_{3}\right)\right)$, so it clearly satisfies C5-C7 when $h_{1}, h_{2}$ do.

\subsection{Equalizer}

Similarly, the construction of equalizers derived in Nets naturally extends to equalizers of labeled nets. With notations of fig. 4, we take $\Lambda=\Lambda_{1}$ for the label set of $\mathcal{N}$, and define the labeling function by $\lambda=\lambda_{1} \circ e$. The morphism $e: \mathcal{N} \rightarrow$ $\mathcal{N}_{1}$ then clearly satisfies $\mathrm{C} 5-\mathrm{C} 7$. For the universal property, the morphism $\psi$ : $\mathcal{N}_{3} \rightarrow \mathcal{N}$ is defined on transitions by $\psi_{T}=e_{T}^{-1} \circ h_{T}$. So $\operatorname{Dom}\left(\psi_{T}\right)=\operatorname{Dom}\left(h_{T}\right)$, and $\psi$ clearly satisfies $\mathrm{C} 5-\mathrm{C} 7$.

\subsection{Pullback}

Assume the $f_{i}: \mathcal{N}_{i} \rightarrow \mathcal{N}_{0}$ are morphisms of labeled nets. The pullback $\mathcal{N}=\mathcal{N}_{1} \wedge$ $\mathcal{N}_{2}$ is defined as follows, by combining the definitions of product and equalizer (section 2).

Transitions. We distinguish "shared" transitions in $\mathcal{N}_{1}$ and $\mathcal{N}_{2}$, i.e. those having an image in $\mathcal{N}_{0}$, from "private" ones, the others. For private transitions, the definition of the pullback mimics the definition of the product. For shared transitions, only pairs that match through the $f_{i}$ are preserved.

$$
\begin{aligned}
T_{s}= & \left\{\left(t_{1}, t_{2}\right) \in T_{1} \times T_{2}: t_{i} \in \operatorname{Dom}\left(f_{i}\right), f_{1}\left(t_{1}\right)=f_{2}\left(t_{2}\right)\right\} \\
T_{p}= & \left\{\left(t_{1}, t_{2}\right) \in T_{1} \times T_{2}: t_{i} \notin \operatorname{Dom}\left(f_{i}\right), \lambda_{1}\left(t_{1}\right)=\lambda_{2}\left(t_{2}\right)\right\} \\
& \cup\left\{\left(t_{1}, \star\right): t_{1} \in T_{1}, t_{1} \notin \operatorname{Dom}\left(f_{1}\right), \lambda_{1}\left(t_{1}\right) \in \Lambda_{1} \backslash \Lambda_{2}\right\} \\
& \cup\left\{\left(\star, t_{2}\right): t_{2} \in T_{2}, t_{2} \notin \operatorname{Dom}\left(f_{2}\right), \lambda_{2}\left(t_{2}\right) \in \Lambda_{2} \backslash \Lambda_{1}\right\} \\
T= & T_{s} \cup T_{p}
\end{aligned}
$$

Notice that the label condition doesn't appear in (11) : it comes as a consequence of $f_{1}\left(t_{1}\right)=f_{2}\left(t_{2}\right)$, since morphisms preserve labels.

Places. Places are obtained by inspecting transitions selected in $T$.

Consider first a private transition $\left(t_{1}, t_{2}\right) \in T_{p}$, where one (at most) of the $t_{i}$ can be $\star$. Assume $p_{i} \rightarrow_{i} t_{i}$ (or equivalently $t_{i} \rightarrow_{i} p_{i}$ ) in $\mathcal{N}_{i}$, with $t_{i} \neq \star$. Observe that necessarily $p_{i} \notin \operatorname{Dom}\left(f_{i}\right)$, otherwise $f_{i}$ would be defined at $t_{i}$. Such a place

\footnotetext{
${ }^{4}$ With the convention that $\psi\left(t_{3}\right)=(\star, \star)$ means "undefined."
} 
$p_{i}$ induces a singleton equivalence class in $P$, either $\left(p_{1}, \star\right)$, or $\left(\star, p_{2}\right)$. We denote by $P_{p}$ all such "private" places.

Consider now a pair of shared transitions $\left(t_{1}, t_{2}\right) \in T_{s}$, where $f_{1}\left(t_{1}\right)=t_{0}=$ $f_{2}\left(t_{2}\right)$. Consider for example a place $p_{1} \in{ }^{\bullet} t_{1}$ (or equivalently $p_{1} \in t_{1} \bullet$, and symmetrically for a place $\left.p_{2} \in \bullet^{\bullet} t_{2}^{\bullet}\right)$.

a. If $p_{1} \notin \operatorname{Dom}\left(f_{1}\right)$, then $\left[\left(p_{1}, \star\right)\right]^{\bullet}\left(t_{1}, t_{2}\right)$ is reduced to $\left(p_{1}, \star\right)$, which yields another private place in $P_{p}$.

b. If $p_{1} \in \operatorname{Dom}\left(f_{1}\right)$, let $p_{0} \in P_{0} \cap \bullet t_{0}$ satisfy $p_{1} \stackrel{f_{1}}{\longleftrightarrow} p_{0}$. By C4 applied to $f_{2}$, there exists $p_{2} \in{ }^{\bullet} t_{2}$ such that $p_{2} \stackrel{f_{2}}{\longleftrightarrow} p_{0}$, so $\left(p_{1}, \star\right) R^{\bullet}\left(t_{1}, t_{2}\right)\left(\star, p_{2}\right)$ in the product $\mathcal{N}_{1} \times \mathcal{N}_{2}$. The resulting equivalence class $\left[\left(p_{1}, \star\right)\right]^{\bullet}\left(t_{1}, t_{2}\right)$, takes the form $\left(Q_{1}, Q_{2}\right)$, with $\emptyset \neq Q_{i} \subseteq P_{i}$, and yields a "shared" place in the pullback.

In summary:

$$
\begin{aligned}
P_{p}= & \left\{\left(p_{1}, \star\right): p_{1} \in P_{1}, p_{1} \notin \operatorname{Dom}\left(f_{1}\right), \exists\left(t_{1}, \cdot\right) \in T, p_{1} \in{ }^{\bullet} t_{1} \bullet\right\} \\
& \cup\left\{\left(\star, p_{2}\right): p_{2} \in P_{2}, p_{2} \notin \operatorname{Dom}\left(f_{2}\right), \exists\left(\cdot, t_{2}\right) \in T, p_{2} \in t^{\bullet}\right\} \\
P_{s}=\left\{\left(Q_{1}, Q_{2}\right): Q_{i} \subseteq P_{i}, Q_{i} \subseteq \operatorname{Dom}\left(f_{i}\right), \exists\left(t_{1}, t_{2}\right) \in T_{s},\right. & \\
& \left.Q_{1} \uplus Q_{2} \text { equiv. class of } \equiv^{\left(t_{1}, t_{2}\right)} \text { or of } \equiv^{\left(t_{1}, t_{2}\right)}\right\} \\
P= & P_{p} \cup P_{s}
\end{aligned}
$$

In (14), the dot in $\left(t_{1}, \cdot\right)$ stands for either $t_{2}$ or $\star$, and symmetrically for the second line.

Initial places. By abuse of notation, let us identify a private place like $\left(p_{1}, \star\right)$ to $\left(Q_{1}, Q_{2}\right)=\left(\left\{p_{1}\right\}, \emptyset\right)$, and $\left(\star, p_{2}\right)$ to $\left(Q_{1}, Q_{2}\right)=\left(\emptyset,\left\{p_{2}\right\}\right)$. So $\left(Q_{1}, Q_{2}\right)$ denotes a general place in $P$.

$$
P^{0}=\left\{\left(Q_{1}, Q_{2}\right) \in P: Q_{1} \subseteq P_{1}^{0}, Q_{2} \subseteq P_{2}^{0}\right\}
$$

Flow. Let $\left(Q_{1}, Q_{2}\right) \in P$ and $\left(t_{1}, t_{2}\right) \in T$ (where one of the $t_{i}$ can be $\star$ ). Then

$$
\begin{aligned}
& \left(Q_{1}, Q_{2}\right) \rightarrow\left(t_{1}, t_{2}\right) \Longleftrightarrow Q_{1} \subseteq \bullet^{\bullet} t_{1} \text { in } \mathcal{N}_{1}, Q_{2} \subseteq{ }^{\bullet} t_{2} \text { in } \mathcal{N}_{2} \\
& \left(t_{1}, t_{2}\right) \rightarrow\left(Q_{1}, Q_{2}\right) \Longleftrightarrow Q_{1} \subseteq t_{1}{ }^{\bullet} \text { in } \mathcal{N}_{1}, \quad Q_{2} \subseteq t_{2} \bullet \text { in } \mathcal{N}_{2}
\end{aligned}
$$

with the convention that $\emptyset \subseteq{ }^{\bullet}$ and $\emptyset \subseteq \star^{\bullet}$ hold.

Morphisms $g_{i}$. Let $\left(t_{1}, t_{2}\right)$ be a transition of $T$, one has $g_{i}\left(t_{1}, t_{2}\right)=t_{i}$ if $t_{i} \neq$ $\star$, and is undefined otherwise. Let $\left(Q_{1}, Q_{2}\right)$ be a general place in $P$, one has $\left(Q_{1}, Q_{2}\right) \stackrel{g_{i}}{\longleftrightarrow} p_{i}$ iff $p_{i} \in Q_{i}$.

\subsection{Special Case}

We examine here the special case where morphisms $f_{i}: \mathcal{N}_{i} \rightarrow \mathcal{N}_{0}$ are partial functions not only on transitions, but also on places (instead of being relations on 
places). The definition changes only for $P_{s}$ in (15) : when place duplications are forbidden, equivalence classes of shared places are reduced to two elements only.

$$
\begin{gathered}
P_{s}=\left\{\left(p_{1}, p_{2}\right): p_{i} \in P_{i} \cap \operatorname{Dom}\left(f_{i}\right), f_{1}\left(p_{1}\right)=f_{2}\left(p_{2}\right)=p_{0},\right. \\
\left.\exists\left(t_{1}, t_{2}\right) \in T_{s}, f_{1}\left(t_{1}\right)=f_{2}\left(t_{2}\right)=t_{0}, p_{0} \in t_{0} \bullet\right\}
\end{gathered}
$$

This definition coincides with the proposition of [9] (and also to an early version of the present notes), apart from the extra condition that places created in (14) and (20) be connected to at least one transition of the pullback. An example is given in fig. 9,
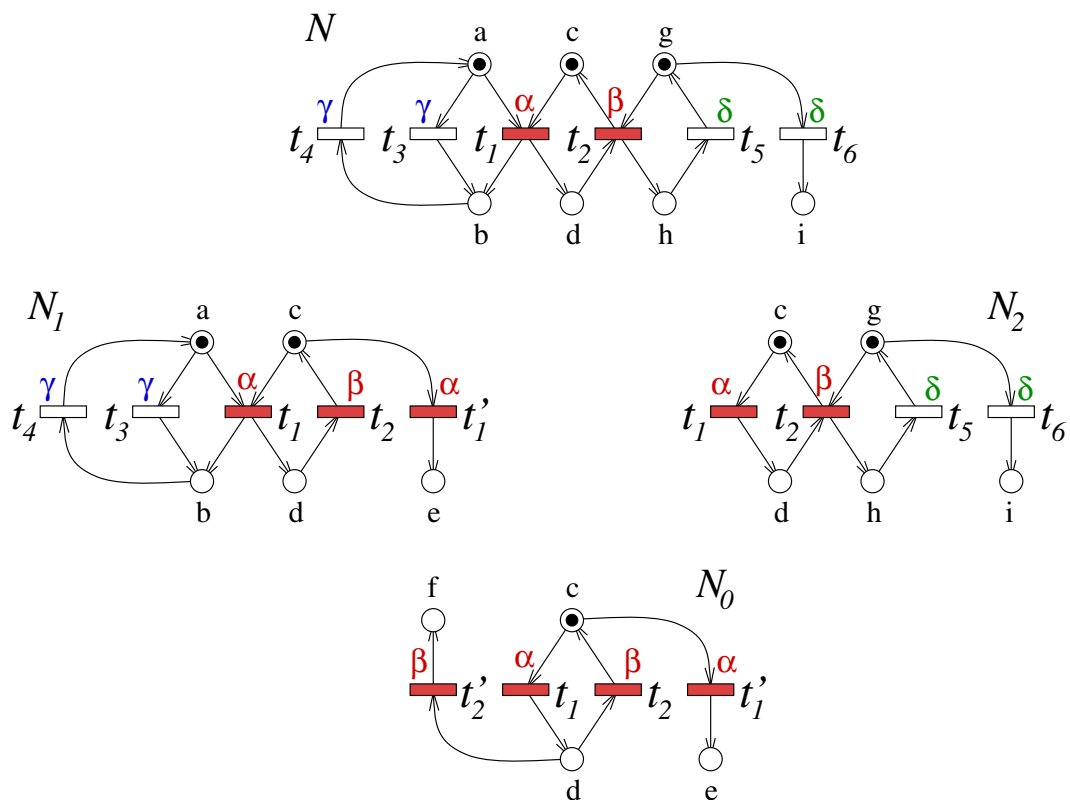

Fig. 9. Example of a pullback: $\mathcal{N}=\mathcal{N}_{1} \stackrel{\mathcal{N}_{0}}{\wedge} \mathcal{N}_{2}$, in the simple case of injective morphisms. Morphisms are represented by common names on transitions and places. Transition labels are indicated by Greek letters. Observe that transition $t_{1}^{\prime}$ of $\mathcal{N}_{1}$ disappears in $\mathcal{N}$ since it finds no partner in $\mathcal{N}_{2}$ with the same image in the interface net $\mathcal{N}_{0}$. This example doesn't reflect the full generality of the pullback construction since outside the domains of $f_{1}$ and $f_{2}$, transitions of $\mathcal{N}_{1}$ and $\mathcal{N}_{2}$ don't synchronize: $\left(\Lambda_{1} \cap \Lambda_{2}\right) \backslash \Lambda_{0}=\emptyset$.

\section{Conclusion}

The original motivation for this work was the derivation of a simple construction for pullbacks of safe nets, thus providing a way to express in a categorical framework the combination of nets that interact by sharing places and transitions. We actually obtained more: we proved the existence of all equalizers in Nets, which, in conjunction with the existence of all products, proves the existence of all (small) limits in Nets. 
Expressing the combination of nets as a categorical limit has some advantages. Consider for example the unfolding operation 4, that associates the unfolding $\mathcal{U}(\mathcal{N})$ to a safe net $\mathcal{N} . \mathcal{U}$ is actually a functor from $N$ ets to the subcategory $O c c$ of occurrence nets, and we know that $\mathcal{U}:$ Nets $\rightarrow O c c$ has a left adjoint, and so preserves limits. As a consequence, when $\mathcal{N}=\mathcal{N}_{1} \wedge \mathcal{N}_{0} \mathcal{N}_{2}$, one immediately obtains $\mathcal{U}(\mathcal{N})=\mathcal{U}\left(\mathcal{N}_{1}\right) \wedge_{O}^{\mathcal{U}\left(\mathcal{N}_{0}\right)} \mathcal{U}\left(\mathcal{N}_{2}\right)$ where $\wedge_{O}$ denotes the pullback in Occ. This result expresses that the factorized form of a net immediately gives rise to a factorized form on runs of this net. Moreover, one obtains for free the existence of pullbacks in $O c c$, with a formal expression for $\wedge_{O}$ : let $\mathcal{O}_{0}, \mathcal{O}_{1}, \mathcal{O}_{2}$ be occurrence nets, one has $\mathcal{O}_{1} \wedge \mathcal{O}_{O} \mathcal{O}_{2} \equiv \mathcal{U}\left(\mathcal{O}_{1} \wedge \mathcal{O}_{0} \mathcal{O}_{2}\right)$, where the last pullback is computed in Nets, and where $\equiv$ means "isomorphic to."

The results above naturally extend to general limits: whatever the way one combines elementary nets to build a larger system (by products, pullbacks, etc.), a similar decomposition holds on the unfolding (or on the trellis 19]) of the global system. We believe this is an important key to study large systems by parts (see 17, 18] for examples of modular diagnosis based on these ideas).

Acknowledgment. The author would like to thank Marek Bednarczyk for fruitful discussions, and Philippe Darondeau for his useful comments.

\section{References}

1. S. Mac Lane: Categories for the Working Mathematician, Springer-Verlag, 1971.

2. G. Winskel : A new Definition of Morphism on Petri Nets, LNCS 166, pp. 140-149, 1984.

3. M. Nielsen, G. Winskel: Petri Nets and Bisimulation, BRICS report no. RS-95-4, Jan. 1995.

4. G. Winskel: Categories of models for concurrency, Seminar on Concurrency, Carnegie-Mellon Univ. (July 1984), LNCS 197, pp. 246-267, 1985.

5. G. Winskel: Event structure semantics of CCS and related languages, LNCS 140, 1982, also as report PB-159, Aarhus Univ., Denmark, April 1983.

6. G. Winskel : Petri Nets, Algebras, Morphisms, and Compositionality , Information and Computation, vol. 72, pp. 197-238, 1987.

7. M.A. Bednarczyk, L. Bernardinello, B. Caillaud, W. Pawlowski, L. Pomello : Modular System Development with Pullbacks, ICATPN 2003, LNCS 2679, pp. 140-160, 2003.

8. M.A. Bednarczyk, A. Borzyszkowski, R. Somla: Finite Completeness of Categories of Petri Nets, Fundamenta Informaticae, vol. 43, no. 1-4, pp. 21-48, 2000.

9. B. Koenig: Parallel Composition and Unfolding of Petri Nets (Including Some Examples), private communication, 2005.

10. K.L. McMillan, Using unfoldings to avoid the state explosion problem in the verification of asynchronous circuits, in Proc. 4th Workshop of Computer Aided Verification, Montreal, 1992, pp. 164-174.

11. J. Esparza, Model checking using net unfoldings, Science of Computer Programming 23, pp. 151-195, 1994.

12. J. Esparza, C. Schröter, Reachability Analysis Using Net Unfoldings, Workshop of Concurrency, Specification and Programming, volume II of Informatik-Bericht 140, pp. 255-270, Humboldt-Universität zu Berlin, 2000. 
13. S. Melzer, S. Römer, Deadlock checking using net unfoldings, CAV'97, LNCS 1254, pp. 352-363, 1997.

14. V. Khomenko, M. Koutny, A. Yakovlev, Detecting State Encoding Conflicts in STG Unfoldings Using SAT, Fundamenta Informaticae, Volume 62, Issue 2, pp. 221-241 IOS Press, 2004 (Special Issue on Best Papers from ACSD 2003).

15. V. Khomenko, M. Koutny, A. Yakovlev, Logic Synthesis for Asynchronous Circuits Based on STG Unfoldings and Incremental SAT, Fundamenta Informaticae, Volume 70, Issue 1-2, pp. 49-73, IOS Press, 2006.

16. A. Benveniste, E. Fabre, S. Haar, C. Jard, Diagnosis of asynchronous discrete event systems, a net unfolding approach, IEEE Trans. on Automatic Control, vol. 48, no. 5, pp. 714-727, May 2003.

17. E. Fabre, A, Benveniste, S. Haar, C. Jard: Distributed Monitoring of Concurrent and Asynchronous Systems, Journal of Discrete Event Systems, special issue, pp. 33-84, May 2005.

18. E. Fabre: Distributed Diagnosis based on Trellis Processes, 44th Conf. on Decision and Control (CDC), Seville, Spain, 12-15 Dec. 2005.

19. E. Fabre, Trellis processes : a compact representation for runs of concurrent systems, INRIA research report, no. RR-5554, March 2005. 